\title{
A Modeling Method of Multimedia Database based on Semantic Sequence
}

\author{
Wenlei Zhang ${ }^{1, \text { a }}$ \\ ${ }^{1}$ The Chinese People's Liberation Army Unit 92493, Dalian, 116023, China \\ aemail: zhangwenlei_HLD@163.com
}

Keywords: Case Relation; Multi-media; Database; MIS

\begin{abstract}
With the development of scientific research and production organization, a large number of audio and video data resources are accumulated in the business system, which is a distinctive feature of scientific research and production. In this paper, a modeling method which extracts semantic sequence from scientific research in the fields of production, through the four tuple structure to describe the relationships between instances, and then implement a more rational database model is proposed. By comparing the current multimedia database construction methods, illustrates the rationality of this method.
\end{abstract}

\section{Introduction}

With the continuous development of scientific research and production organization, the organization has accumulated a large number of visual audio and video data, which plays an important role in the relevant fields [1]. Although the current radio and television industry has built a mature media asset management system [2], but due to the particularity of scientific research and production organizations, these systems are difficult to meet the demand [3].

In this paper, a modeling method which extracts semantic sequence from scientific research in the fields of production, through the four tuple structure to describe the relationships between instances, and then implement a more rational database model is proposed. By comparing the current multimedia database construction methods, illustrates the rationality of this method.

\section{Present Situation Analysis}

At present, solve multimedia query scheme mainly has the following kinds: a scheme to the main mission, establishing the task file management system, multimedia information as part of archives information, dependent on them; two of the program is to be arranged, according to the type of media display entities were built; scheme three directly to the media as the main line, not to classification and direct manual extraction of some key fields of media, a media information table.

These three methods have advantages and disadvantages: attachment to the media program database task as the main line, for the task of display is complete, the general also has the time axis relatively complete, can show the sequence relationship between the media, and to a certain extent, can show the causal relationship between the media and the mutual dependence of it; the disadvantage is that the media is not close relationship between tasks, similar to the lack of correlation between the media or the same type of media, it is difficult to make a transverse retrieval and comparison [4]. The type of media to show support to the entity database, its advantage is that it can have a more comprehensive understanding of the same entity type, retrieval obvious advantage and comparative advantage, and can increase the task field associated with the task, to avoid missing the first method; however, it must be to establish a table for each type of entities such as missiles, artillery and so on, which requires very professional knowledge, but also the media involved in a very wide scope, it is difficult to guarantee each form the reasonable and relatively complete [5]. The third scheme is relatively flexible, the utility model has the advantages of database is easy, can quickly enter the practical stage, but the extraction of the key field is not objective, also often leads to information loss, the retrieval capability is relatively weak for the first 
two programs.

As the analysis shows, the above scheme has its advantages and disadvantages, relatively speaking, third kinds of scheme is easy to implement, but its search ability is weak in practical relevance; the first scheme pays too much attention to the media information and task, explore the media information point lost in the face, often lead to lateral are not closely linked. It is difficult to find the value of the media; second schemes than the other two schemes, there are certain advantages, but relates to the field of broad professional knowledge for database designers demanding, in short, is difficult to establish a data table, but the table does not meet the requirements.

Therefore, it is urgent to design a new kind of multimedia database to solve the problem.

\section{Solution Proposal}

The relation between an instance and an instance in a semantic sequence is represented by an instance of $M$ four tuple structure.

$M=\left(I,\{P\},\left\{P_{\text {condition }}\right\},\left\{\left(I^{\prime},\left\{P^{\prime}\right\},\left\{P^{\prime}\right.\right.\right.\right.$ conditon $\left.\left.\left.\},\left\{\left(I^{\prime},\left\{P^{\prime \prime}\right\},\left\{P^{\prime \prime}{ }_{\text {condition }}\right\},\{\ldots\}\right)\right\}\right)\right\}\right)$.

$I$ represents an instance of the central concept class in the "central concept sequence" of the semantic sequence. $P$ and $P_{\text {condition }}$ represent the name of the property owned by the instance. In instances of knowledge reasoning stage, $P_{\text {condition }}$ involved in reasoning the attribute name, is to judge the case is in accordance with the attribute name for inference access standards; in the instantiation stage, $P$ from attribute description sequence acquisition, is obtained from the center of the concept of sequence. Sequence element "sequence of constraint conditions" or the properties of the sequence is described to "may contain sub semantic sequence", in which $I$ is obtained, and is represented by I'. $P^{\prime}$ represents which is involved in the reasoning part of the corresponding attribute name. $P^{\prime}$ conditon is to judge whether $I$ ' meets the standards for admittance into reasoning property name. Similar situation agrees to the $I$ ', $P$ "', $P$ " condition, corresponding definitions. When there is no "conditional constraint sequence" and "attribute description sequence" in the semantic sequence, the structure of the semantic sequence obtained is : $M=\left(I,\{P\},\left\{P_{\text {condition }}\right\},\{\Phi\}\right)$.

The () in four tuple structure $M$ represents one of the elements constituting a multi group structure. \{\} represents the elements of the set. The form of the four tuple structure is described by standard: $M=\left(I,\{P\},\left\{P_{\text {condition }}\right\},\left\{\mathrm{M}^{\prime}\right\}\right)$.

For example, the semantic sequence "in / Radar cross-sectional area / /200/ square meter / condition / under /, / maximum detection distance / /500/ km /." is presented in instance four tube through the construction method above:

instance of $M=$ (individual of OntClass "Maximum detection range", ...

\{OntPropertyname "Numerical data" $\},\{\Phi\}, \ldots$

\{(individual of OntClass “Radar cross section”, ...

\{OntPropertyname "Numerical data” $\},\{\Phi\})\}$ ).

\section{Database structure design}

In this way, the database structure design can be effectively guided:

The database model is divided into three layers , the first layer is the basic information layer, including the basic information table of the media, the full text table, the media table and the auxiliary information table, as is shown in Figure 1.

The second layer is a temporary sub surface layer, which is set up according to the set conditions.

The third layer is the sub surface layer, when the temporary sub table is stable, it can be changed into a sub table. 


\section{Design of basic information layer}

This layer is a relatively independent category, can be regarded as an independent MIS system, the purpose is to allow the system to put into use as soon as possible. The media table stores the media information, such as photos, videos, sound files, because the file is generally relatively large, so a separate table; the relevant information media basic information table stores the media, such as the name, time and so on; the media text table to store the cited media and the necessary media content, each corresponding to the media the content of a single. As for the auxiliary information table is refers to, such as user table, user records and some other concerned in the content.

This layer has the function of independent design, with the basic information of the media as the main line, the design of query and statistical functions. To achieve more easily, but also to achieve this layer, you can deliver the use, without having to complete the development of the system is completed.

The development of this layer is equivalent to third kinds of solutions, in order to avoid the defects of the third schemes, the design of the following two layers.

\section{Basic information layer}

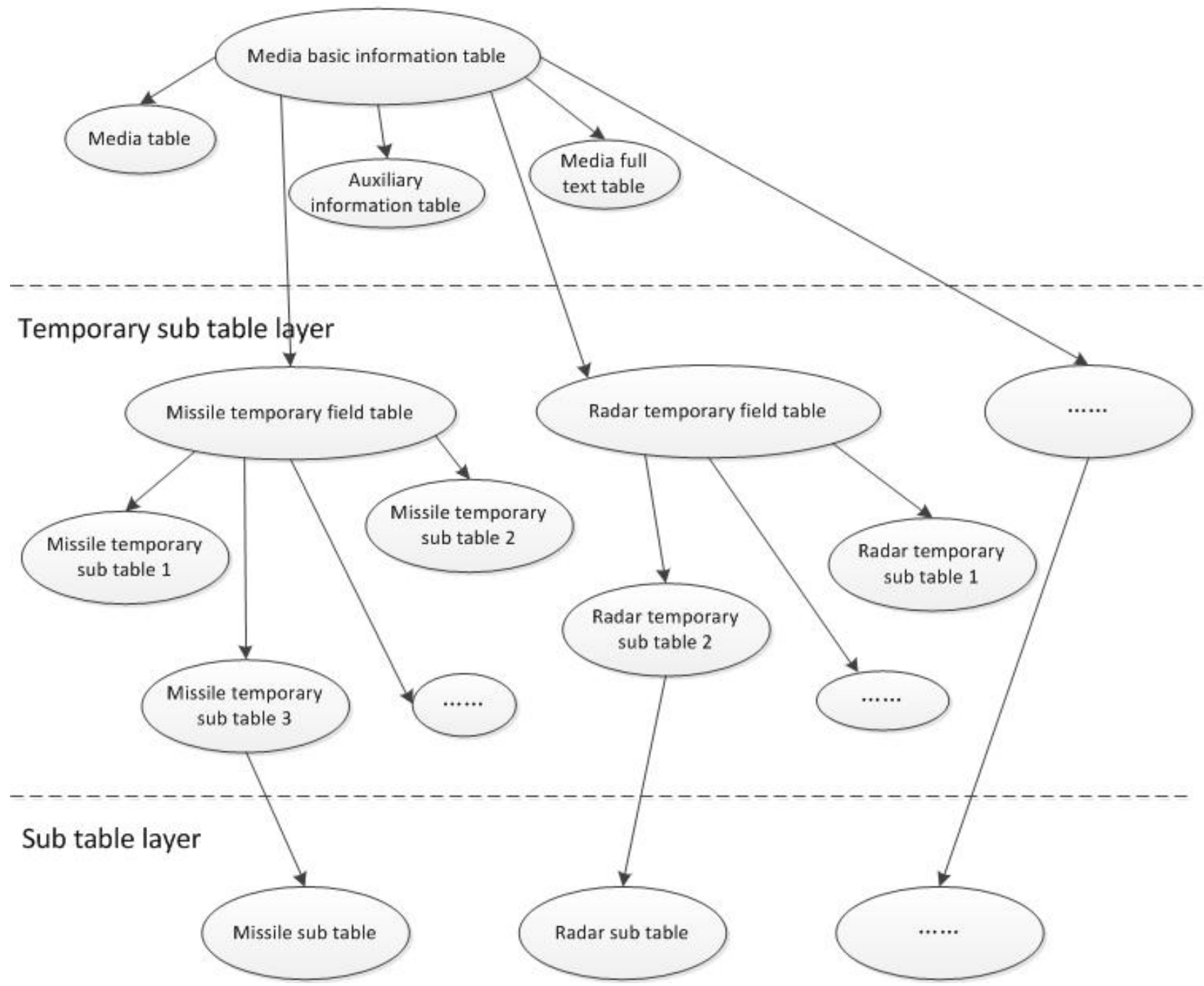

Fig.1. Three layers structures

\section{Design of temporary sub table layer}

When a kind of media is necessary to separate into a class, it is necessary to design a temporary sub table.

If the missile is required to be a separate tab, it is necessary to establish a temporary table below. Temporary table of missile, missile temporary sub table 1 , missile temporary sub table $2 \ldots . . .$. The 
missile temporary field list contains the field and the field type for the missile, and the field type can only be two types: character type and numeric type. Each missile temporary sub table corresponds to each field in the missile temporary field list. That is, each field is a single table. The reason for this design is the fault tolerance of the consideration, if the establishment of each field is not properly, it will only affect the field itself, will not cause a greater impact on the system.

\section{Method comparison and analysis}

This method combines the advantages of second and three, which can be quickly put into use, and with the use of the system, and with the second program to be able to lateral alignment, retrieval ability. At the same time to avoid the defects of the two, due to the temporary layer of buffer design, improper selection of the field will not cause a greater impact on the system. The main advantages of this scheme are as follows:

(1) The establishment of the database can quickly enter the practical stage as soon as possible;

(2) With the in-depth use of database, can continue to improve;

(3) There are more closely linked to similar data, comparison and analysis;

(4) The database developer's domain knowledge is relatively low;

(5) The structure will not change the database cause data loss and other serious consequences.

This program also has certain defects:

(1) The program design is more complex, relative to the general MIS system, the system of the high degree of automation, high requirements for programmers;

(2) The actual application in the sub surface before entering the system, the retrieval efficiency is not high;

(3) The system users use the system, than the general MIS system use personnel responsibility more, also need to bear the growth of system tasks and higher requirements;

(4) The system with high redundancy.

\section{Conclusion}

In this paper, a modeling method which extracts semantic sequence from scientific research in the fields of production, through the four tuple structure to describe the relationships between instances, and then implement a more rational database model is proposed. By comparing the current multimedia database construction methods, illustrates the rationality of this method. After the implementation of the system, the use of the situation, basically achieved the design objectives of the system. In addition, the project provides a way to build a MIS system for the future, and makes a useful attempt.

\section{References}

[1] Yang ZhiPing, Feng Sumei. Metadata storage and retrieval of multimedia database. Journal of Chongqing Teachers College: Natural Science Edition [J]. 2002, 19(1): 14-18.

[2] Gong Zhiguo, Zhou Longxiang. Distributed multimedia database system. Journal of software[J]. 2000, 11(1): 40-48.

[3] Xiao Jianyu, Zhang Dafang. The key technology of multimedia database: multimedia data model. Computer engineering and Applications[J]. 2002, 38(7): 215-217.

[4] Fu Xitian, Sun Jingfu. The key technology of multimedia database management system. Telecommunication Science[J]. 1998, 14(8): 1-4.

[5] Chai Xingwu, Chen Yunsheng. Analysis of the hierarchical structure of multimedia database management system. Computer Science[J]. 1995, 22(1): 61-64. 